\title{
Chronische Krankheiten und der Wirt - Gesundung am Beispiel von Patienten mit Multipler Sklerose
}

\author{
Johannes G. Schmidt \\ Stiftung Paracelsus heute, Einsiedeln, Schweiz
}

\section{Schlüsselwörter}

Chronische Krankheiten · Wirt · Kranksein/Krankheitssituation . Multiple Sklerose · Gesundungsvermögen · Salutogenese · Nosologie $\cdot$ Komplementärmedizin · Altchinesische Medizin

\section{Zusammenfassung}

Chronische Krankheiten sind zuerst oft der Ausdruck von Gesundungsvorgängen, welche die Oberhand gewinnen wollen. In der Altchinesischen Medizin wird der Mensch mit seinen spezifischen Schwächen als Wirt der Krankheit gestärkt, und der verbesserte Wirtszustand ermöglicht dann die Wende von einem klinisch ungünstigen zu einem klinisch gutartigen Verlauf. Bei Patienten mit Multipler Sklerose (MS) scheint der Schub ein Aufräum-Phänomen zu sein, wobei der Körper spontan Kälte und Nässe aus dem vitalen Zentrum wegbewegt. MS-Patienten zeigen in der Regel ein Syndrom aus lang dauernden Verdauungsstörungen, Schlafstörungen, Frieren und/oder Hitze, bei dem dann passager neurale Schädigungen auftreten. Wird das Gesundungsvermögen gestärkt - und nicht die Krankheit «kaltgestellt» -, kann der Körper die innere Kälte und Nässe unter anderem durch Schübe allmählich eliminieren und die Gesundheit wiederherstellen. Mit einem konsequenten Blick hin zur Gesundheit des ganzen Wirts und mit der Beschreibung und Klassifikation salutogener Prozesse könnte die Komplementärmedizin - vielleicht zusammen mit der Hausarztmedizin - ihre wichtige Rolle bekommen.

\section{Keywords}

Chronic disease $\cdot$ Host $\cdot$ Illness $\cdot$ Multiple sclerosis .

Restoring capacity for health · Salutogenesis · Nosology ·

Complementary medicine $\cdot$ Ancient Chinese Medicine

\section{Summary}

The Relation between Chronic Diseases and Their Host -

Clearing Pathogenic Factors in the Case of Patients with Multiple Sclerosis Chronic diseases are primarily the result of the struggle of the human constitution to clear pathogenic factors from the system. In Ancient Chinese Medicine, the aim of the doctor is to strengthen the entire patient, giving specific constitutional weaknesses special attention. As a result of the patient's improved resistance, the disease can turn from a clinically unfavourable to a clinically favourable course. In the case of multiple sclerosis (MS), relapses are spontaneous attempts

\section{KARGER}

Fax +497614520714 Information@Karger.com www.karger.com of the organism to rid itself of cold and damp in its vital centre. As a rule, patients with MS have long-standing digestive disorders, sleep disturbance, and problems of feeling cold and/or hot, which may eventually result in transient neural damages. If the doctor supports the attempts the body makes to eliminate inner cold and damp by way of these relapses, rather than 'freezing' this process, it is possible that health can be restored. The contribution of complementary medicine - perhaps together with family medicine - is to address the health of the host as a whole and to describe and classify the salutogenic processes which are able to restore health.

\section{Mots clés}

Maladies chroniques · Hôte · Malade/Situation de maladie · Sclérose en plaques · Capacité de guérison · Salutogénèse · Nosologie . Médecine complémentaire · Médecine traditionnelle chinoise

\section{Résumé}

Les maladies chroniques et l'hôte: guérison à l'exemple des patients atteints de sclérose en plaques

Les maladies chroniques sont d'abord souvent l'expression de processus de guérison qui veulent prendre le dessus. Dans la médecine traditionnelle chinoise, l'homme, avec ses faiblesses spécifiques est fortifié en tant qu'hôte de la maladie. Et l'amélioration de l'état de I'hôte permet ensuite de passer d'une évolution clinique défavorable à une évolution clinique bénigne. Chez les patients atteints de sclérose en plaque (SEP), la poussée semble être un phénomène de nettoyage où le corps débarrasse spontanément le centre vital du froid et de I'humidité. Les patients SEP présentent en général un syndrome de troubles digestifs, de troubles du sommeil, de sensations de froid/ et ou de chaud de longue durée, au cours duquel apparaissent des lésions nerveuses passagères. Si la capacité de guérison est renforcée et que la maladie ne reste pas «figée» pas, le corps peut éliminer peu à peu le froid et l'humidité intérieure par des poussées entre autres et recouvrer la santé. En ne regardant résolument que la santé de l'hôte entier, et avec la description et la classification des processus salutogéniques, la médecine complémentaire pourrait jouer un rôle important, peut-être en collaboration avec la médecine généraliste. 


\section{Einführung: Gesundung und}

das Gesundungsvermögen

Krankheitsverläufe können im Einzelfall sehr stark vom Durchschnitt abweichen, da eine übliche Diagnose kaum etwas über den Patienten aussagt. Verschiedene Patienten als Wirt derselben Krankheit können entweder chronische Beeinträchtigungen entwickeln und gar einen vorzeitigen Tod erleiden oder aber völlig gesund bleiben (z.B. Brust-, Prostata- und Hautkrebs, Multiple Sklerose (MS) und viele andere mehr). Pathogene Einflüsse treffen auf Wirte mit unterschiedlichem Gesundungsvermögen, und Krankheiten spiegeln die Auseinandersetzung zwischen salutogenen und pathogenen Prozessen wider. Im Grunde sind chronische Krankheiten oftmals Ausdruck von Gesundungsvorgängen, die (mühsam) die Oberhand zu gewinnen versuchen.

Eine Gesetzmässigkeit der Gesundung scheint zu sein, dass Personen in vielen Fällen erst durch den Gesundungsvorgang krank werden. Als Prototyp dieses Phänomens kann man die Grippe betrachten. Findet der Wehrvorgang kompetent statt, dann geht er einher mit Fieber, Schwitzen, Müdigkeit und Krankheitsgefühl. Personen mit mangelhaftem Wei Qi (Wehrkraft der chinesischen Medizin) reagieren hingegen nicht auf Erkältungen und bleiben oberflächlich betrachtet «gesund», wobei die Kälte eindringt und früher oder später irgendwo das Qi und das Xüe («Kraft und Saft» der Chinesischen Medizin) blockiert. Paradoxerweise wird also der gesund Funktionierende krank und der krankhaft (bzw. nicht gesund) Funktionierende bleibt «gesund». Paradox ist auch die Wirkung von Kälte: Sie blockiert und bremst die Zirkulation und führt so zu Hitze und zu Entzündungen. Hitze ist also nicht einfach der Ausdruck von pathogener Hitze, sondern entsteht klinisch viel häufiger aus pathogener Kälte. Auch da gibt es den Prototyp: Eine Frostbeule sieht am Schluss genau gleich aus wie eine Brandwunde; beides wird deshalb in einer phänomenologisch orientierten Klassifikation als Dermatitis bezeichnet. Die Altchinesische Medizin bezeichnet die aus der Kälte entstandene Entzündung als «falsche Hitze» und sucht in der Anamnese akribisch nach der Unterscheidung. Denn das therapeutische Kühlen von Entzündungshitze, das in der Komplementärmedizin fast so verbreitet ist wie in der Schulmedizin, ist eine Sackgasse [1]. Falsche Hitze muss mit Wärme behandelt werden, wobei der entsprechende Yang-Aufbau Monate oder Jahre dauern kann (Yang = im klinischen Kontext Lebensfeuer und Geist - vs. Säfte und Körpermaterie, als Yin bezeichnet) [2].

In der klinischen Praxis lässt sich das Gesundungsvermögen des Patienten durch eine detaillierte Anamnese und aufgrund des klinischen Eindrucks gut erkennen, auch wenn kein einziger Labortest dafür zur Verfügung steht: Welche Erkrankungen sind vorgekommen und wie ist der Patient damit zurechtgekommen? Wie funktionieren die Verdauung und die Mitte/Milz (Bezeichnung für den Funktionskreis der Mitte/Erde in der chinesischen Medizin, der nicht der schulmedizinischen Milz entspricht. Auch die Begriffe Niere, Herz und Lunge werden hier im chinesischen Sinn gebraucht)? Kann die Mitte alles zusammenhalten oder verliert der Patient Blut, Urin, die Form (z.B. Hernien oder Varizen) oder fehlt das Selbstbewusstsein? Kann sich die Person gut entscheiden und Sorgen hinter sich lassen? Wie funktioniert die Oberfläche im Bereich Lunge, Nase, Hals, Haut und Enddarm? Kann sich die Person abgrenzen gegen Pollen, Erreger, Wind und Wetter und gegen Attacken auf der psychischen Ebene oder treten gehäuft Lungen- und Hautprobleme oder «dünnhäutige» Reaktionen auf? Kann der Patient richtig trauern und Verletzungen hinter sich lassen und eingedrungene Pathogene etwa durch gesundes Schwitzen wieder eliminieren? Sind die Grundkräfte ausreichend, halten warm und stützen? Sind diese so warm, dass kein Frieren, kein Bettnässen, keine Nykturie und keine Schlafstörungen auftreten? Sind sie so stark, dass der Rücken gut gestützt ist und nicht schmerzt und die Knochen und Zähne halten? Ist der Geist wach und schützt vor Unfällen oder übertriebenem Lampenfieber, und sieht der Geist klar, um nicht Wunschdenken, falschem Mitleid, falschen Ängsten, einem Opferbewusstsein oder Verschwörungstheorien zu erliegen? Ist ein gesundes, flexibles Durchsetzungsvermögen ohne übertriebene Sturheit vorhanden oder zeigen sich hypochondrische Schmerzen oder Gallenprobleme oder muskuläre und sonstige Verspannungen mit Druckgefühl im Magen oder am Hals? Wo haben sich Schwächen bereits bei der Geburt oder in der Kindheit gezeigt, und wo hat es angefangen?

Wer genaue Anamnesen dieser Art jahrelang übt, wird immer genauer erkennen, wie verschieden Patienten funktionieren und wie gut oder mangelhaft das individuelle Gesundungsvermögen ausgestattet ist.

Wie Abbildung 1 darstellt, ist das Kranksein nicht einfach Folge einer Krankheit, sondern das Resultat aus Krankheit und Wirtszustand - und Umwelteinflüssen. Entscheidend ist das Wechselspiel zwischen pathogenem Angriff und salutogenem Widerstand. Deshalb ist man mit einer Krankheit nicht schon krank (und ohne Krankheit ist man nicht unbedingt gesund). Während sich in der Abbildung das Kranksein/die Krankheitssituation aus der Schnittmenge ergibt (dreifach schraffiert), sind pathologische Befunde (pathologische paraklinische Daten und pathologische klinische Daten) in ihren Differenzmengen Zustände, die nicht schon zum Kranksein führen (einfach und zweifach schraffiert). Eine gute Medizin sollte deshalb Diagnosen nur dann stellen und behandeln, wenn diese das wirkliche Problem des Patienten ausmachen. Überdiagnose ist jedoch eine wachsende Nebenerscheinung der heutigen Medizin [3]. Deshalb stellt sich bei den konven- 
Demographische Daten: Umweltbelastung, Klima, Beschäftigungslage/Arbeitslosigkeit, Religionen/ Konfliktkultur, Bildungswesen etc.

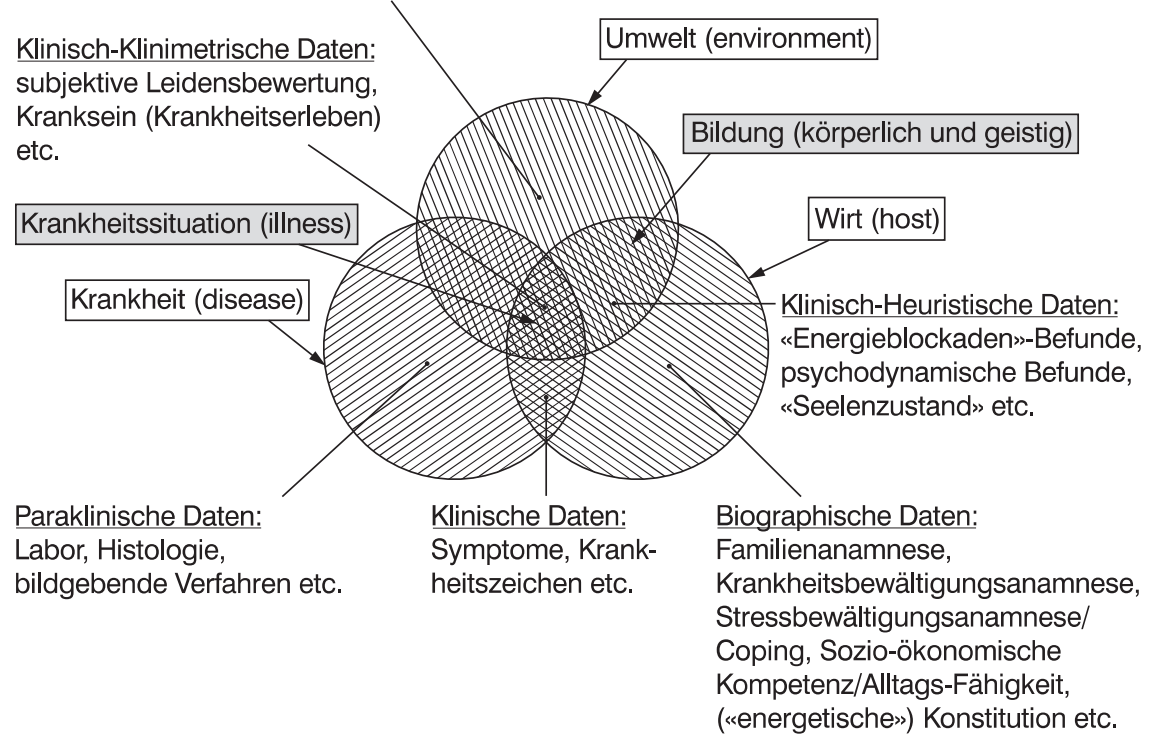

Abb. 1. Kranksein/Krankheitssituation als Produkt von Krankheit (disease), Wirt (host) und Umwelt (environment) [9].

tionellen Diagnosen gemäss ICD (International Classification of Diseases) immer die Frage, ob sie das relevante Problem anzeigen oder ob sie vielmehr nur zu einer unnötigen oder irrelevanten Therapie verleiten. (Die ICD ist eine rein pathologieorientierte Klassifikation. Seit gut 30 Jahren existiert eine International Classification of Impairments, Disabilities and Handicaps (ICIDH), die vor gut 10 Jahren zur International Classification of Functioning, Disability and Health (ICF) weiterentwickelt worden ist. Diese Anstrengungen, Gesundheit und Fähigkeiten des Wirts einzubeziehen, sind in der Praxis bisher folgenlos geblieben.)

In der Altchinesischen Medizin stehen das Gesundungsvermögen und die Widerstandskräfte ganz im Zentrum des prophylaktischen und therapeutischen Vorgehens. Nicht die Krankheit und ihre Symptome werden in erster Linie behandelt oder bekämpft, sondern es wird der Wirt, der ganze Mensch mit seinen spezifischen Schwächen, gestärkt. Angesichts der Bedeutung des Wirts und seines Gesundungsvermögens und angesichts der starken Variation von Krankheitsverläufen wird verständlich, weshalb die Altchinesische Medizin in vielen Fällen in der Lage ist, chronisch-progressive und langsam progrediente, auch maligne Erkrankungen zu stabilisieren und dem Patienten zur Gesundung zu verhelfen. Überraschenderweise persistiert die Pathologie dabei sehr oft und wird auch im morphologischen oder biochemischen Ausmass nicht unbedingt kleiner; der verbesserte Wirtszustand ermöglicht aber die Wende von einem klinisch ungünstigen zu einem klinisch gutartigen Verlauf. Die Medizin hatte ja beispielsweise schon gelernt, dass schlimme oder gutartige Tuberkulose-Verläufe nicht durch pathogene Variationen beim Tuberkulose-Bakterium bestimmt werden, sondern durch die variierenden Widerstandskräfte der Patienten.
Diese waren generell durch die Überwindung von Mangelernährung und feuchtkalten Wohnverhältnissen und individuell etwa durch Kuren in voralpiner Höhe verbessert worden.

Der gutartige Verlauf wird also nicht unbedingt durch das Verschwinden von Pathologien und Pathogenen bestimmt, sondern vielleicht vorwiegend durch das Gesundungsvermögen. Dass eine Bedingung für Gesundheit die Abwesenheit von Krankheiten und Pathologien sei, widerspricht allen klinischen Erfahrungen und allen epidemiologischen Daten, wenn wir einen Moment lang darüber nachdenken. Gesundheit ist vielmehr ein gesundes Reagieren des Wirtes auf Schädigungen, d.h. ein intaktes Gesundungsvermögen. Gesunder Schlaf, gesunde Verdauung, gesunde innere Wärme, gesundes Trauern, gesunde Depressionen usw. und letztlich auch das erfüllte Sterben gehören alle zu einer intakten Gesundheit.

\section{Die moderne Epidemie: Kälteschädigungen aufgrund von Yang-Erschöpfung}

Die alten Chinesen waren sich wie die Alten in der Bibel und in anderen Überlieferungen der stetigen Gefahr des natürlichen Yang-Verlustes beim Menschen und der daraus folgenden Kälteschädigungen bewusst - Yang ist das Licht des Lebens, das dank der ununterbrochenen Überlieferung der asiatischen Medizin von Generation zu Generation auch in seinen klinischen Ausdrucksformen geläufig geblieben ist. Beschrieben ist diese altchinesische Klinik insbesondere im Klassiker Shang Han Lun von Zhang Zhongjing (150-219 n. Chr.), auf Deutsch «Abhandlung über die Kälteschädigungen». Dieses Werk behandelt die 
entsprechenden klinischen Erscheinungen mit den dazugehörigen Rezepturen, wobei das Buch für uns recht kryptisch daherkommt und ohne Unterweisung schwer zu verstehen ist. Ich habe meine Praxis von Hin Chung Got (geb. 1946) gelernt. Er ist Schüler von Tang Buqi (19172004), der die Schriften von Zheng Qin-An (1824-1911) zur Feuerlehre herausgegeben hat.

Kälteschädigungen erfolgen (heutzutage) mehr aufgrund eines Mangels an Lebenswärme und Innerem Feuer (Yang-Mangel) und weniger aufgrund einer starken Einwirkung von pathogener Kälte. Gesunde Organe leisten ein stetes Aufräumen eingedrungener pathogener Einflüsse wie der Kälte. Angeborene Mängel können aber eine Funktionsschwäche von Lunge, Mitte und Niere bedingen, sodass die Elimination von Erkältungen und Kälte durch Fieber und Schwitzen (Lunge) oder über den Stuhlgang (Mitte) und andere Wege (Urin, Schleimhäute, Haut usw.) nicht richtig erfolgt. Hat die Niere zu wenig Feuer, dann fehlt das Yang überall. Das Nieren-Yang kann genetisch von Geburt, aber häufig auch durch Überanstrengung und Auszehrung im Laufe des Lebens geschwächt sein - wenn «es an die Niere gegangen» ist. Ein Übermass an pathogener Kälte, die auf Yang-Leere trifft, kann z.B. zu einer Multiplen Sklerose (MS) führen. Sie kann aber auch Polyarthritis, chronische Erkältungen, Colitis ulcerosa, chronische Schmerzen, Schlafstörungen, Depressionen, Stoffwechselstörungen, Morbus Basedow, Abwehrschwäche mit der Folge von Krebs, Borreliose, Polio usw. usw. ermöglichen oder auslösen; es kommt auf die genetischen und erworbenen Schwächen an.

Die altchinesische Behandlung zielt wie gesagt nicht in erster Linie auf das Pathogen ab, sondern auf die körperlich-seelische Verfassung, die nicht in der Lage war, mit dem Pathogen zurechtzukommen und dieses wieder zu eliminieren oder schachmatt zu setzen. Entscheidend ist deshalb die Diagnose der individuellen Kräfte-Architektur mit ihren Grundschwächen. Die Kräuter-Strategien des Shang Han Lun stützen den Körper, seine Gesundungsarbeit zu Ende zu bringen und seine angeborenen Grundschwächen «auszuwachsen». In vielen Fällen zeigt sich, dass angeborene Grundschwächen - vielleicht gerade in der Auseinandersetzung mit Krankheiten - sich auflösen bzw. «erlösen». Es geht in der Praxis folglich nicht in erster Linie um ein Bekämpfen des Leidens, sondern um eine Stützung der Lebenskräfte, damit der Patient durch das Leiden hindurch kommt, seine Schwächen (und seine falschen Ängste) erkennt und so gesund wird. Erkennen und Wahrnehmen erfolgt durch eine Verbesserung des Yang und des $Q i$ in den von Schwächen betroffenen Organkreisen - es braucht eine ausreichende "Beleuchtung», um sehen zu können, wie man ist. Diese strategische Sicht oder Vision des Arztes, die er durch Erfahrung und durch die Führung durch einen kundigen Lehrer gewinnt, ist für den Verlauf wahrscheinlich sehr entscheidend. Der Arzt darf nicht aus falschem Mitleid mit dem Patienten zusammen dem produktiven Teil des Leidens ausweichen und so die Genesung behindern. Er muss den Patienten trösten und ihm mit seiner klaren Sicht auf die sich abzeichnende Entwicklung die Ängste nehmen, was dann möglich ist, wenn er aus viel Erfahrung seiner Sache sicher ist. Das klingt kompliziert, und es ist auch anspruchsvoll; neben den schwierigen gibt es aber immer auch einfachere Fälle.

Kälteblockaden und Kälteschädigungen sind meist vergesellschaftet mit Nässe- oder Schleimstau oder Qioder Xüe-Stagnation oder gelegentlich mit Hitze oder Wind, die durch Turbulenzen Stagnation erzeugen. Es ist immer wichtig zu erkennen, wie und wo es angefangen hat. Wind kann aus Hitze entstehen, Hitze aus Stagnation und Stagnation aus Yang-Mangel (die Pferde sind müde und kraftlos, deshalb stagniert der Karren im Dreck - oder: mit einem zu schwachen Motor läuft ein Auto heiss). Dann hilft nur geduldiger Yang-Aufbau. Die Ausleitung von Wind, Schleim oder "Giften» hingegen ermöglicht vielleicht etwas Symptomen-Kosmetik, ist letztlich aber nutzlos und schwächt; primär ist der Yang-Aufbau notwendig. Yang-Aufbau macht aber auch lebendig und reaktiv, und Symptome können deshalb zunehmen. Es ist ganz entscheidend, dann den Yang-Aufbau zu erkennen und im Blick zu behalten und sich nicht an Symptomen aufzuhalten, seien es auch die (vermeintlichen) Leitsymptome der Krankheit. Hat der Körper etwas Yang übrig, will er immer aufräumen und erzeugt dadurch Krankheitssymptome. Deshalb sind viele Krankheiten zuerst einmal Gesundungsversuche, die der Körper spontan unternimmt, um pathogene Einflüsse wieder loszuwerden. Scheitert er chronisch bei diesem Versuch, dann entsteht eine chronische Krankheit und führt zu immer mehr Beeinträchtigung. Gelingt es aber, das Yang und die Kräfte zu halten und wieder aufzubauen, dann räumt der Körper immer wieder auf, wenn gerade genug Yang da ist und das Wetter und die Jahreszeit günstig sind. Wenn wir aber dem Zeitgeist folgen, das Yang nicht bewahren und Krankheitsäusserungen zunehmend «kaltstellen», dann tritt eine Epidemie von Kälteschädigungen auf, wie z.B. eine $\mathrm{Zu}$ nahme von MS.

\section{Patienten mit Multipler Sklerose}

Bei der MS ist der schubweise Verlauf interessant. Sorgfältige klinische Beobachtung und gute Verläufe bei MSPatienten weisen darauf hin, dass es nicht entscheidend ist, Schübe zu verhindern. Entscheidend ist die Fähigkeit des Patienten, Schübe wieder überwinden zu können. Denn der Schub gehört zu den klinisch vielfältigen AufräumPhänomenen, die der Körper bei genügender Gesundungskraft spontan beginnt. Und so bricht die als MS bezeich- 
nete Krankheit aus, wenn der Körper Kälte/Nässe aus dem (vitalen) Zentrum wegbewegt. Kälte und Nässe, die natürlich schon lange vor dem Ausbruch der Krankheit vorhanden waren, sieht man an der blassen Zunge mit Zahnabdrücken. Der «Ausbruch» der MS ist also ein Gesundungsvorgang (die pathogene Kälte muss ausbrechen, weil das Feuer des Yang sie verdrängt). Denn wenn das Zentrum entlastet wird, kann der Körper die vital wichtige Kräfteregeneration wieder besser bewerkstelligen, auch wenn Nässe und Kälte mehr oder weniger lange in der Peripherie liegen bleiben können und die Funktion der Nerven beeinträchtigen. Nässe verursacht Taubheit, während Kälte sich zu falscher Hitze wandelt und für Entzündungen sorgt. In den meisten Fällen verschwinden bei MS-Patienten die Symptome der ersten Schübe wieder vollständig, weil der Körper noch in der Lage ist, auch in der Peripherie wieder aufzuräumen und den Gesundungsimpuls zu Ende zu führen. In aller Regel wird das Yang in der heutigen Zeit aber nicht bewahrt, und das Ausmass der pathogenen Belastung durch Nässe und Kälte im Zentrum ist entsprechend grösser, als dass ein einziger Schub zur vollständigen Räumung der Kälte/Nässe-Belastung und zur restitutio führen könnte.

MS verläuft in einigen Fällen klinisch sehr gutartig und verschwindet mehr oder weniger häufig sogar wieder ganz durch eine Spontanremission. In anderen Fällen kann MS sehr aggressiv und schnell verlaufen und zum Tod führen. Wenn wir nicht die MS, sondern den Wirt behandeln, kann es uns gelingen, einen ungünstigen Verlauf in einen gutartigen Verlauf überzuführen. Wenn wir MS-Patienten genau befragen und untersuchen, dann finden wir immer Verdauungsprobleme - seit langer Zeit oder gar seit der Kindheit. Dünner Stuhlgang, Blähungen und unangenehmer Bauch, manchmal träger Stuhlgang bis zu tagelangem Stuhlverhalten. Oft besteht auch eine Unfähigkeit zum Schwitzen und Fiebermachen. Und wir finden oft Schlafschwierigkeiten und das Greisensymptom Nykturie in jungen Jahren. Die Kälte stört den Schlaf und die Blase. Kortison-Behandlungen führen oft zur Zunahme von Schlafstörungen, denn Kortison ist ein potentes Yin-Mittel, das falsche Hitze und damit MS-Symptome beseitigen kann, aber die pathogene Kältebelastung und den YangMangel verstärkt. Wir finden fast immer Frieren, kalte Füsse oder unangenehme Hitzegefühle, die sich mit dem Frieren abwechseln oder es übertünchen (falsche Hitze, die aus Kälte entsteht). Wenn eine Behandlung dann zum Wechsel von heissen Füssen zu kalten Füssen führt, ist das ein erster Schritt in die richtige Richtung, auf den wir unter Umständen einige Monate bis ein Jahr warten müssen. Schlaf- und Verdauungsstörungen bedeuten, dass KräfteRegeneration und Kräfte-Erhalt kompromittiert sind, sodass der Körper der pathogenen Kälte und der Krankheit immer weniger entgegensetzen kann. Deshalb ist es strategisch entscheidend, die eigentlichen MS-Symptome hintanzustellen und zunächst Verdauung und Schlaf sowie das Frieren in Ordnung zu bringen. Warme Organe funktionieren besser und ein warmer Körper ist besser durchblutet, kann besser verdauen und besser den Schlaf finden und somit wieder regenerieren.

Wie gesagt sind Schübe Ausdruck davon, dass der Körper noch in der Lage ist, die Kältebelastung aus dem Zentrum wegzuschieben. Schübe sind ein Vitalitätszeichen. Deshalb ist die nur geringe und klinisch irrelevante Schubprophylaxe, die mit MS-Medikamenten möglich ist, eine Pyrrhus-Medizin. Interferon kennt als Nebenwirkungen ein Grippesyndrom, Anorexie und Verdauungsstörungen, Schlafstörungen, Kopfschmerzen usw. An diesen Nebenwirkungen erkennt man die Wirkung von Kälte. Mit Interferon wird also die noch vorhandene Vitalität so «kaltgestellt», dass weniger Schübe erfolgen können, was einen fatalen Verlauf am Ende wahrscheinlich beschleunigt. Die wichtigen Regenerationsmotoren wie Verdauung und Schlaf werden beeinträchtigt. Die Zahl der Schübe ist kein valides Erfolgskriterium; geeignet sind erst die längerfristige Lebensqualität mit den dazugehörigen Fähigkeiten der Mobilität sowie Schlaf, Verdauung, innere Ruhe, Lebensfreude und allenfalls die Mortalität. Systematische Analysen in seriösen Reviews (Cochrane Collaboration) kommen denn auch zu dem Ergebnis, die vorliegenden Studien seien nicht in der Lage, einen Nutzen von Interferon zu dokumentieren [4]. Trotzdem wird Interferon heute den Patienten von den Neurologen aufgedrängt, weil der hilflose Helfer-Arzt und der Zeitgeist offenbar Strohhalm-Medizin verlangen, die der Auseinandersetzung mit Vergänglichkeit und Sterben aus dem Weg geht, obwohl diese Auseinandersetzung heilsam wäre. Gut $20000 \mathrm{CHF}$ kostet das nebenwirkungsträchtige und letztlich nutzlose Medikament pro Jahr. Interessant ist, dass MS-Patienten sich gelegentlich gegenseitig von einer Interferon-Behandlung abraten, weil sie spüren, dass es ihnen nicht guttut.

\section{Zwei klinische Fallbeispiele}

Methodisch gesehen sind Fallbeispiele klinischer Verläufe im Grunde recht aussagekräftig, denn bei chronischstabilen Krankheiten kann der Patient als seine eigene Kontrolle dienen - ähnlich dem «N-of-1-Trial» - und stellt so eine besondere Form der kontrollierten Studie dar [5]. Mittlerweile sind es etwa 20 MS-Patienten, die sich bis zu 15 Jahren in meiner Behandlung befinden. Gewiss ist dies eine Selektion von Personen, die ihr Vertrauen in diese Art der Behandlung gesetzt haben, sodass eine Verallgemeinerung auf alle MS-Patienten nicht ohne Weiteres gemacht werden kann. Wichtig ist die Betrachtung der individuellen Gegebenheiten, die unterschiedliche Behandlungen mit unterschiedlichen Rezepturen erfordern. Jeder MSPatient benötigt ein anderes Rezept, das seiner individuel- 
len «Kräfte-Architektur» entspricht. Auf der anderen Seite wird das gleiche oder gleichartige Rezept für gleichartige Patienten verwendet, die verschiedene Krankheiten haben - z.B. Heuschnupfen, Polyarthritis, Rückenschmerzen, MS, Schlafstörungen usw.

Eine wichtige Rolle im Verlauf spielt das Bewusstsein im Sinne der Selbstwahrnehmung. Vor allem der Zustand des Herzens, das als Kaiser der Organe gilt, bestimmt, wieweit der Geist wach ist und die Dinge richtig sieht. Ist ein Mensch, der nicht schwimmen kann, ins Wasser gefallen, dann hat er die Chance, schnell schwimmen zu lernen und sich zu retten. Ob er böswillig ins Wasser geschubst worden ist oder ob er selber hineingefallen ist, ändert nichts an dieser Chance. Das geschubste Opfer hat es aber wahrscheinlich schwerer, weil sein Bewusstsein, Opfer zu sein, die Hilfe von aussen sucht - und nicht findet und nur wertvolle Zeit verliert. Der Andere kann sich hingegen direkt auf die Rettung durch seine eigene Hilfe besinnen. Der beherzte Geist bestimmt, ob das Bewusstsein diese Chance sieht oder ob diese im Dunkeln bleibt. Ein Mensch, der nicht (beherzt) sieht, wird seine Kräfte falsch einsetzen und sein Yang erschöpfen - und mit der Zeit Belastungen wie der Kälte nicht mehr widerstehen können. So wird jemand krank - bis er durch die Krankheit vielleicht die Gesundung findet. Bis zu einem gewissen Grad ist es immer nötig, dass ein Patient sein eigener Arzt wird, indem er sich verstehen lernt und sieht, was er braucht und dass er nicht gegen seine Kräfte leben kann. Vielleicht aus diesem Grund ist die Behandlung der Mitte/Milz in jeder Rezeptur ein zentraler Bestandteil. Die Mitte/Milz ist das Organ der Vermittlung und des Ausgleichs und ermöglicht die "Herz-Nieren-Verbindung», die sozusagen Herz und Verstand zusammenbringt. In der natürlichen Gesundung wird das Herz oft zunehmend heftig "erschüttert», damit es offenbar so die Fühler ausstreckt und sich bei ausreichender Mitte/Milz-Kapazität «am rechten Fleck» binden kann. Wenn der Arzt eine richtige Rezeptur verwendet, gelingt es früher oder später, dass der Patient mehr «zu sich kommt», sich erkennen lernt und sein eigener Arzt werden kann - falls der Himmel hilft und nicht Verhängnis und Unglück das Yang zu sehr erschöpfen.

Ich bin jeweils froh, wenn ein Patient von einer Interferon-Therapie Abstand nimmt, weil Interferon die Gesundung hindert und weil diese Therapie auch ein Zeichen sein kann, dass Ängstlichkeit und nicht Beherztheit die Wahrnehmung des Patienten bestimmt. Aber auch eine zu mutige Ablehnung des Interferons kann Zeichen einer Herz-Schwäche sein. Man darf nicht dogmatisch sein, denn oft führt der einzig gangbare Weg über einen Umweg.

Die Bewusstseinsentwicklungen sind sehr subtil und nicht nur von der Beherztheit (vom Herzen), sondern von allen Organen auf ihre Weise bestimmt. Auch die körperlichen Äusserungen sind oft sehr subtil und beinhalten alle möglichen Verbesserungen und Verschlechterungen, doch wenn die regenerativen Funktionen wie Schlaf und gute Verdauung im Sinne guter Nahrungsverwertung trotz zeitweiliger Rückfälle in Richtung Besserung zeigen, dann ist der Verlauf gut. Zunge, Puls und Ausstrahlung bestätigen ebenfalls die Gesundungsstrasse.

\section{Fall 1}

Eine 29-jährige Patientin ist seit 3 Jahren in meiner Behandlung. Die ersten MS-Symptome sind mit 23 aufgetreten - Gefühllosigkeit in den Fingerspitzen, später Sehstörungen. Frühgeburt, schon lange kalte Hände und Füsse, braucht Bettsocken. Gelegentlich Bettnässen als grösseres Kind. Lieber Sommer als Winter. Schlecht schwitzen, nie Fieber ausser einmal im Alter von 10 Jahren mit Fieberphantasien. Mittelohrentzündungen. Rückenschmerzen und Hexenschuss mit ca. 20 Jahren. Stuhlgang öfters breiig. Heisshunger. Schlaf als Kleinkind gestört, Albträume, zu den Eltern ins Bett. Mensblutungen eher schwach. Tiefer Blutdruck und Orthostase-Schwindel. Zunge: Eher blass, Zahnabdrücke, Delle an der Spitze. Puls: Hat Widerstand (Kraft). Ein Arztbrief fasst die Situation zusammen:

«Die Patientin hat mich aufgesucht, primär mit der Frage nach dem Nutzen einer Interferon-Therapie. Interferon führt wahrscheinlich zu einer kleinen Reduktion von Schüben unter einer Zunahme gewisser Nebenwirkungen über den Zeitraum von 2 Jahren, aber es ist unklar, ob diese Wirkung klinisch wirklich relevant ist und längerfristig anhält. Nur ‘wahrscheinlich), weil in den entsprechenden Studien gewisse methodische Kriterien (Studien-Dropout) nicht zuverlässig erfüllt sind. ...

... Das heisst wahrscheinlich, dass mit Interferon die MS-Symptome ein bisschen «kaltgestellt〉 werden, was den Organismus aber in die Sackgasse treibt. Wir kennen das auch bei der peri-/postmenopausalen Östrogen-Behandlung, die zwar oft wirksam die Symptome verbessert, aber am Schluss zu einer Mortalitätserhöhung führt. Auch da wird 〈falsche Hitze〉 mehr oder weniger wirksam gekühlt, der Organismus aber in seiner Widerstandsfähigkeit geschwächt.

... Bei dieser Patientin betrachte ich die Voraussetzungen als günstig, weil sie ein richtiges und nicht perverses Spüren hat (sie schützt sich vor Kälte und sucht nicht dauernd die Kühlung der falschen Hitze, wie man das oft sehen kann). Es ist gut möglich, dass die MS bei ihr ganz verschwinden wird. Wie die deutlichen Zahnabdrücke zeigen, wird es aber 2-3 oder noch mehr Jahre dauern, bis Nässe und Kälte überwunden sind.

... Die MS-Symptome sind zweitrangig und zuerst ja einmal ein Heilungsversuch bzw. -vorgang. Die passagere Taubheit in den Fingern bedeutete, dass der Körper noch gut in der Lage war, Nässe herauszubewegen, die dann eine Zeit lang in den Fingern hängen geblieben ist. Primär entwickeln sich die Widerstandskräfte, wenn die Verdauung in Ordnung kommt (geformter Stuhlgang) und das Frieren verschwindet und das Wei Qi in die Lage kommt, richtig zu schwitzen und Fieber zu machen und Erkältungen zu eliminieren. Dann wird die Grundpathologie von Nässe und Kälte, die zu Schüben und MS-Symptomen führen 
kann, schwinden. Und allenfalls lädierte Nerven könnten sich wieder langsam erholen.

Ich habe der Patientin ein entsprechendes Kräuterrezept empfohlen und abgegeben (Ge Gen Tang-Modifikation). Ich halte es auch für wichtig, dass von November bis Februar jeweils keine Blutentnahmen erfolgen, und von Eisen-Kosmetik und entsprechenden Infusionen habe ich abgeraten. Betreffend Ernährung sollte der Yang-Aufbau im Vordergrund stehen. Dazu ist Fleisch (vor allem Lamm, Huhn, Rind, Wild, Raubfische, Crevetten) besonders geeignet und lang gekochte Brühen ...»

Die Rezeptur - über die Zeit in der Dosierung leicht verändert - sieht heute so aus: $15 \mathrm{~g}$ Ma Huang, $40 \mathrm{~g}$ Gui Zhi, 20 g Rou Gui, 15 g Gan Cao, 75 g Fu Zi, 60 g Bai Zhu, $40 \mathrm{~g}$ Fu Ling, $100 \mathrm{~g}$ Huang Qi, $20 \mathrm{~g}$ Dang Gui. Also handelt es sich um eine Erkältungsrezeptur, die das Schwitzen aufbaut, mit Mitteln gegen Kälte und Nässe und Mitteln zum Blutaufbau. Das Schwitzen ist im Sommer 2012 praktisch normal geworden; kein roter Kopf mehr. Warme Füsse, weniger Frieren. Stuhlgang oft noch breiig, immer guter Appetit, Gewicht abgenommen (= Kälte wird über den Stuhlgang wegbewegt, wobei ein guter Appetit ohne Heisshunger besteht, Verdampfen von Kälte und Nässe hat zu Gewichtsabnahme geführt). Die Zunge hat mehr Farbe, ist aber noch tendenziell blass, hat noch Zahnabdrücke und ist noch etwas belegt. Im heissen August 2012 ist noch einmal ein heftiger Schub mit Taubheitsgefühlen an den unteren Extremitäten bis zur Bauchnabelhöhe steigend aufgetreten. Eine Nacht lang auch Störungen der Blase mit Harndrang. Mit diesem Schub ist hingegen der Schlaf besser geworden, was zeigt, dass die schlafstörende Kälte im Zentrum abgenommen hat. Und die Beherztheit hat sich nach einer kurzen Erschütterung durch Ängste, die durch die früheren neurologischen Belehrungen geprägt worden sind, gefestigt. Nach 2 Monaten waren alle Taubheitsgefühle und Parästhesien wieder verschwunden - bis heute.

Dieser Verlauf sieht vielversprechend aus und mein klinischer Eindruck sagt, dass die MS überwunden ist. Für den guten Verlauf wichtig scheint dabei das adäquate Wahrnehmen und Verhalten der Patientin zu sein, welche die wohltuende Wirkung einer Yang-aufbauenden Ernährung spürt und ebenso gespürt hat, dass Vitamine und Power-Drinks, die sie gut gemeint bekommen hat, ihre Verdauung stören und im Bauch aufliegen (Vitamine und Power-Drinks sind Yin-lastig und entsprechend kraftlos). Zur Konsolidierung und Überwindung der ganzen NässeKälte-Belastung sind noch Kräuter über zwei bis drei Winter nötig - und natürlich eine gute Ernährung und ein vernünftiges Regenerationsverhalten.

\section{Fall 2}

Ein 60-jähriger Patient ist seit 14 Jahren in Behandlung mit altchinesischen Kräutern; die Diagnose MS erfolgte vor 20 Jahren. In den ersten 6 Jahren nach der Diagnose er- folgte eine stetige Krankheitsprogression bis hin zu invalidisierenden Gehstörungen, Blasenfunktionsstörungen, Dauerschwindel sowie Appetit- und Schlafstörungen. Früher immer gesund, als 15-Jähriger starke Angina. Unter der altchinesischen Kräutertherapie hat sich die Krankheit stabilisiert; keine Progression mehr, Appetit besser, seltener Schwindel und etwas besserer Schlaf. Allerdings konnten die peripheren Paresen und anderen Beeinträchtigungen nicht alle überwunden werden, und die Verdauung ist noch immer sehr träge. Markant verbessert hat sich die Lebensfreude; Gelassenheit hat die ehemals vorherrschenden Gefühle von Wut, Ärger und Ungeduld abgelöst. Die Kräutertherapie wurde durch eine prozessorientierte Akupressur begleitet, was die Orientierung weg von einer kräftezehrenden Denkweise unterstützt hat. Bei derart schweren Fällen sind die psychischen Veränderungen oft das wichtigste klinische Zeichen.

Dieser Fall hätte ohne unsere Therapie fast sicher einen weit ungünstigeren (chronisch progressiven) Verlauf genommen, was die Wirkung der Therapie in diesem Einzelfall quasi beweist, auch wenn methodisch korrekt natürlich eine unabhängige und eventuell verblindete Beurteilung erfolgen müsste.

Die ersten Jahre wurde wechselweise mit einer Variation von Wen Pi Tang (20 g Da Huang, $30 \mathrm{~g}$ Dang Shen, $45 \mathrm{~g}$ Fu $\mathrm{Zi}, 30 \mathrm{~g}$ Gan Cao, $30 \mathrm{~g}$ Gan Jiang) und mit einer Modifikation von Shen Fu Tang plus Dang Gui Bu Xüe Tang (30 g Dang Shen, 60 g Fu Zi, 20 g Dang Gui, 15 g Gan Cao, 100 g Huang Qi, 15 g Ma Huang) behandelt. Die grosse Stagnation der Mitte aufgrund der Yang-Erschöpfung ist hier der Angelpunkt; die Stagnation führte zeitweise auch zu schwarzem Zungenbelag («Verkohlung» bei Stau-Hitze). Heute besteht die Behandlung aus einer Variation von Ge Gen Tang-Fuzi Li Zhong Wan mit kräftig Fu Zi (30 g Ge Gen, 20 g Ma Huang, 250 g Fu Zi, 80 g Bai Zhu, $50 \mathrm{~g}$ Fu Ling, $30 \mathrm{~g}$ Gan Cao, $50 \mathrm{~g}$ Liang Jiang, $50 \mathrm{~g}$ Dang Shen).

\section{Diskussion}

Chronische, unheilbare Krankheiten lassen sich besser verstehen, wenn das individuell variierende Gesundungsvermögen des Patienten genau betrachtet wird und wenn wir den Wirt der Krankheit erkennen und behandeln. MSPatienten zeigen in der Regel ein Syndrom aus lang dauernden Verdauungsstörungen, Schlafstörungen, Frieren und/oder Hitze usw., bei dem in der Folge neurale Schädigungen auftreten - zuerst passager und dann dauerhaft. Wenn wir die neurologische Störung als das zentrale Element der Krankheit betrachten, besteht die Gefahr einer $\mathrm{zu}$ reduktionistischen Sicht auf Therapieziele, die falsche «Erfolge» vortäuschen. Wenn wir aber die unspezifischen Störungen von Verdauung, Schlaf und Lebenswärme als 
die zentralen Merkmale bei Patienten mit MS verstehen, die eher als peripheres Detail auch passagere neurologische Störungen bekommen, dann können viele MS-Patienten gesund werden.

Der 2001 verstorbene Yale-Professor Alvan Feinstein hat als sorgfältiger schulmedizinischer Internist und als Klinischer Epidemiologe schon vor 20 Jahren eine Nosologie des Wirtes als Ergänzung zur ICD-Klassifikation gefordert [6]. Es leuchtet unmittelbar ein, dass sich klinische Praxis und klinische Forschung also konsequent die Frage stellen müssen: Wie geht es dem ganzen Wirt unter der Intervention (also nicht nur der Krankheit und ihren (neurologischen) Symptomen)? Um auf diese Weise eine sensitivere Nosologie und wirksamere Therapien für den Wirt (und nicht nur gegen Krankheiten) zu entwickeln, kann das klinische Wissen entsprechender komplementärmedizinischer Richtungen eine entscheidende Grundlage bilden. Die daraus folgende klinische Forschung ist innovativ und erfordert Querdenken (Wirt statt Krankheit) und wird durch die heute gültigen methodischen Grundlagen (evidenzbasierte Medizin) prinzipiell schon vorgezeichnet. In der Tat hat die seriöse Schulmedizin in der Cochrane Collaboration eine MS-Schub-Reduktion nach 2 Jahren als ungeeignetes klinisches Erfolgskriterium erkannt und den Surrogat-Trugschluss hinter der Interferon-«Wirksamkeit» geltend gemacht [4]. Surrogat-Wirkungen wie günstig aussehende biochemische oder pathologische Veränderungen gelten in der real existierenden Schulmedizin (und Komplementärmedizin?) zwar noch oft als der Beweis für Wirksamkeit und Nutzen, doch dieser Trugschluss wird sich nicht ewig halten. Wenn sich die einfache Logik durchsetzt, dass auch der Wirt und sein Gesundungsvermögen und nicht so sehr pathologische Veränderungen die Gesundheit bestimmen, wird das falsche Surrogat-Denken seine Vormacht verlieren und einer effektiveren und pluralistischeren Medizin Platz machen.

So ist denn die massgebliche Frage für die weitere $\mathrm{Zu}$ kunft der Medizin nicht die der Wirksamkeit, sondern jene, was eine gesunde und erwünschte Wirkung ist und was nicht. Wird der Wirt gesund oder wird nur eine einzelne Krankheitsäusserung (kosmetisch) verbessert? Kennen wir die längerfristigen Auswirkungen auf die Gesamtmorbidität und auf die Gesamtmortalität? Wie können wir Gesundung und das Gesundungsvermögen definieren und diagnostizieren statt nur die Krankheit? Die sorgfältige Beobachtung in der Praxis stellt dabei die wichtigste Erkenntnismethode dar. Am Schluss lässt sich ja nur mit den einfachen Mitteln der Anamnese und der klinischen Verlaufsbeobachtung erkennen (vielleicht in der Form der Klinimetrie [7]), was der Patient von der Therapie hat und wie sich seine Gesundheit und seine Fähigkeiten insgesamt verbessern und verändern - ohne Täuschung durch einen Surrogat-Trugschluss.

Die kontraproduktive Verwendung «wirksamer» Mittel gegen salutogene Prozesse sollten die Komplementärmedizin und insbesondere auch die Hausarztmedizin als Trugschluss erkennen können, indem sie entsprechend forschen und sich schulen, die Gesamtgesundheit und das Wechselspiel zwischen Krankheiten und Gesundungsvermögen genügend zu verstehen. Die zum unüberlegten Reflex gewordene Behandlung aller Pathologien ist uferlos, weil stets neue diagnostische Möglichkeiten und Risikofaktoren dazukommen. Die Menschen werden dadurch nicht gesünder, sondern immer häufiger durch iatrogene Überdiagnose und Übertherapie beeinträchtigt $[3,8]$. Mit einem konsequenten Blick hin zur Gesundheit des ganzen Wirtes und mit der Beschreibung und Klassifikation salutogener Prozesse wird die Komplementärmedizin - zusammen mit der Hausarztmedizin - ihre wichtige Rolle bekommen.

\section{Disclosure Statement}

Der Autor hat für diesen Beitrag keinerlei Art von Unterstützungen erhalten. Ein Interessenkonflikt besteht nicht.

\section{Literatur}

1 Schmidt JG: Gemüse gegen Fleisch, Altchinesische Ernährungslehre und Yang Mangel - eine Analyse statistischer Trug schlüsse in einer Ernährungsstudie. Schweiz Z Ganzheitsmed 2011;23:349-355.

$\checkmark 2$ Schmidt JG, Bühlmann S, Shao Ying: Ist die Aristolochiasäure in der Asarum-Pflanze tatsächlich kanzerogen? Die wichtige Be deutung epidemiologischer Effektmodifi kation - mit einem klinischen Fallbeispiel Schweiz Z Ganzheitsmed 2012;24:242-248.

3 Welch HG, Schwartz L, Woloshin Steve: Overdiagnosed: Making People Sick in the Pursuit of Health. Beacon Press, 2012.
4 Rice GP, Incorvaia B, Munari L, Ebers G, Polman C, D’Amico R, Filippini G: Interferon in relapsing-remitting multiple sclerosis. Cochrane Database Syst Rev 2001;(4): CD002002.

5 Abel U, Koch A (eds): Non-Randomized Comparative Clinical Studies. Düsseldorf, Symposium Publishing, 1998.

6 Feinstein AR: Why do we need clinical epidemiology? - A practice-oriented clinical science; in Schmidt JG, Steele RE (eds): Kritik der medizinischen Vernunft: Schritte zu einer zeitgemässen Medizin - Ein Lesebuch. Mainz, Kirchheim, 1994, pp 233-243.

7 Feinstein AR: Clinimetrics. New Haven, CT, Yale University Press, 1987.
8 Schmidt JG: The epidemiology of mass breast cancer screening: a plea for a valid measure of benefit. J Clin Epidemiol 1990; 43:215-225.

9 Schmidt JG: Die Vision einer pragmatischen klinischen Forschung oder das Ende der Diskussion über «Placebo» und «spezifische Wirkungen»; in Schmidt JG (ed): Placebo Wertvoll wenn es dem Patienten nützt? Methodologie einer nutzenorientierten, pragmatischen klinischen Forschung. Placebo - Valuable if it helps the patient? Methods and Design of a Pragmatic Clinical Research, Oriented towards Patient Benefit. Forsch Komplementärmed 1998;5(suppl 1): 102-111. 